\title{
Reform and Practice of Learning Evaluation of Programming Courses under Mixed Teaching Mode
}

\author{
Ding Linhua ${ }^{1, a,{ }^{*}}$, Liu Li ${ }^{1, b}$, Wang Jiuru ${ }^{1, c}$ and Wang Xiaojie ${ }^{1, d}$ \\ ${ }^{1}$ School of Information Science and Engineering, Linyi University, Linyi, ShanDong, China \\ aprivate_ding@163.com, bliuli@lyu.edu.cn, cwangjiuru@lyu.edu.cn, dwangxiaojie@lyu.edu.cn \\ *Ding Linhua
}

Keywords: Mixed Teaching Mode, Programming Courses, Learning Evaluation.

\begin{abstract}
The programming courses is an important basic course for computer science. The MOOC/SPOC wave and information teaching tools promote the exploration and practice of mixed teaching mode. The change of the teaching mode will inevitably bring about the change of the student evaluation system. The programming courses needs to clarify the course objectives, and adopt the evaluation system with diversifying the evaluation objectives, diversifying the evaluation items, and diversifying the evaluation subjects.
\end{abstract}

\section{混合教学模式下程序设计类课程学习评价改革与实践}

丁林花 ${ }^{1, a}$, 刘丽 ${ }^{1, b,{ }^{*}}$, 王九如 ${ }^{1, c}$, 王晓洁 $1, \mathrm{~d}$

1临沂大学信息科学与工程学院, 临沂, 山东, 中国

aprivate_ding@163.com, bliuli@lyu.edu.cn, wangjiuru@lyu.edu.cn, dwangxiaojie@lyu.edu.cn

*丁林花

关键词：混合教学模式;程序设计类课程; 学习评价

中文摘要. 程序设计类课程是计算机类专业的重要基础课程, MOOC/SPOC浪潮和信息化教学 工具推动了混合教学模式的探索和实践。教学模式的改变必然带来学生学习评价体系的变革, 程序设计类课程需要明确课程目标, 采用评价目标多样化、评价项目多样化、评价主体多元 化的学习评价方案。

\section{1. 引言}

程序设计类课程是计算机专业的核心课程重要组成,该课程群包括《程序设计语言基础》、 《数据结构》、《算法分析与设计》等, 从程序语言基础开始培养学生会编程, 到数据结构 培养学生会根据实际问题设计高效的算法、会写好的程序, 再到算法分析与设计研究复杂算 法和工程问题。程序设计类课程群充分展现了学生从获取知识、应用知识到创新知识的层次 化递进式提高进程。随着MOOC/SPOC的浪潮和新一代信息技术的快速发展, 程序设计类课 程也开展了混合式教学模式的探索和实践, 传统的学生评价体系也随之改革, 成绩的构成不 再是一锤定音, 整个学习周期内的表现都应该纳入最终成绩构成。 


\section{2. 程序设计类课程混合教学模式改革}

截至到目前, 程序设计类课程经历了三个阶段的改革: 第一个阶段理论为主、实验为辅, 主要时间花费在了教师的理论讲解上, 学生的实际编程能力薄弱, 更谈不上实际应用和创新 应用; 第二个阶段理论实践并重, 从教师讲转变到以学生学为中心，虽然编程实践加强了， 但是学生仍然是在单机环境非网络平台上进行编程训练, 难以设计和实现逻辑严密的、效率 高的算法和程序; 第三个阶段, 课程群将在线评测系统引入到了教学中, 学生所有的编程实 践都采用线上形式在该平台完成，通过大量编程实践提高学生的编程能力和实际应用能力。 虽然经过以上改革历程, 程序设计类课程教学水平逐步发展, 教学质量逐步提高, 学生能力 也逐步提升, 但是目前的教学模式仍然满足不了学生的个性化学习、自主学习和协同学习的 需求。

长期的教学实践发现, 对于程序设计类课程每个学生的学习基础和学习能力差异很大。 传统的一刀切的课堂教学模式会造成基础好想早跑的学生没有快跑的平台和资源, 而基础差 跑不动的学生也没有弥补的平台和资源, 小规模的在线课程SPOC为个性化教学提供了可能。 同时程序设计能力的提高和实际解决问题的能力培养则需要大量的编程训练, 在线评测系统 作为SPOC课程的有效补充和实验平台可以取得非常好的效果。雨课堂是清华大学联合学堂在 线共同研发的移动互联网教学工具，受到当代互联网土著一族的极大欢迎。

本课程群积极探索和实践 “SPOC+在线评测+雨课堂”的混合教学模式, SPOC提供线上视 频, 在线评测系统提供线上编程练习, 雨课堂课内课外都可以进行课件推送、习题测试、学 习互动等。在这种“课内课外、线上线下、移动固定”互融互补的多样化混合式教学模式下, 需要制定新的学生过程性评价体系。

\section{3. 学生学习过程性评价体系}

\section{1 考核方式及具体要求}

以《C 语言程序设计》课程为例, 学生学习评价采用过程性评价体系, 包括平时成绩和 期末考试成绩两部分成绩。

其中期末考试成绩占 $50 \%$ ，在考核 $\mathrm{C}$ 语言相关基础知识掌握程度的基础上，重点考核理 论知识的应用能力, 以及解决相关复杂工程问题的能力。期末考试采用机试闭卷的方式进行 考核，题目包含函数题、编程题和简答题。既有涉及基础知识的考核题目，又有综合能力测 试的题目, 充分考核了基础知识的掌握程度、学生分析问题、解决问题的能力与程序设计方 法的灵活运用能力。

平时成绩占 $50 \%$ ，由单元测试成绩，平时测试成绩以及小组作业成绩组成和平时表现加 分组成。其中, 单元测试成绩占平时成绩的 $40 \%$, 由两次单元测试平均分组成, 一次在期中 时间点进行，一次在期末时间点进行，分别考察学生前半段和后半段对课程基础知识的掌握 和灵活运用能力。平时测试成绩占平时成绩的 $40 \%$, 主要采用每周测试的形式考察学生对于 每一周知识点的掌握程度和灵活运用能力, 根据课程进度和学生掌握程度一周测试一次或者 两周测试一次。小组作业占平时成绩的 $20 \%$, 主要考核学生解决复杂工程问题的能力, 培养 学生系统化、模块化、量化分析等核心专业意识。平时表现加分主要依据课堂表现、课下答 疑情况, 最高加5 分。

\section{2 成绩评定办法及依据}

课程考核学生对 $\mathrm{C}$ 语言和程序设计掌握和使用情况、运用所学知识解决相关工程问题的 能力, 支持本课程设计的教学目标, 全面反映本课程对本专业毕业要求达成情况的支撑关系。 
表1 考核方式及成绩评定办法

\begin{tabular}{|c|c|c|c|}
\hline 课程目标 & 考核方式/占比 & 考核内容与方法 & 成绩评定指标与依据 \\
\hline \multirow{4}{*}{$\begin{array}{l}\text { 目标1. 掌握 C 语言 } \\
\text { 顺序结构、分支结构、 } \\
\text { 循环结构、数组、字符 } \\
\text { 串、结构体等基本语 } \\
\text { 法, 掌握程序设计思想 } \\
\text { 和方法, 并能够运用于 } \\
\text { 解决复杂工程问题 }\end{array}$} & 考试50\% & $\begin{array}{c}\text { 课程相关知识点掌 } \\
\text { 握程度 }\end{array}$ & 试题评分标准 \\
\hline & 单元测试20\% & $\begin{array}{c}\text { 期中单元测试和期 } \\
\text { 末单元测试 }\end{array}$ & PTA测试评分标准 \\
\hline & 平时测试20\% & 每周测试 & PTA测试评分标准 \\
\hline & 小组作业10\% & $\begin{array}{c}\text { 学生分组完成的大 } \\
\text { 作业 }\end{array}$ & 小组作业评分标准 \\
\hline \multirow{4}{*}{$\begin{array}{l}\text { 目标2. 能够使用函数 } \\
\text { 进行模块化编程, 能够 } \\
\text { 分析程序时间效率, 能 } \\
\text { 够分析同一问题不同 } \\
\text { 实现的时间差距, 具有 } \\
\text { 系统化、模块化、量化 } \\
\text { 分析等核心专业意识 }\end{array}$} & 考试10\% & $\begin{array}{c}\text { 课程相关知识点掌 } \\
\text { 握程度 }\end{array}$ & 试题评分标准 \\
\hline & 单元测试10\% & $\begin{array}{c}\text { 期中单元测试和期 } \\
\text { 末单元测试 }\end{array}$ & PTA测试评分标准 \\
\hline & 平时测试 $20 \%$ & 每周测试 & PTA测试评分标准 \\
\hline & 小组作业60\% & $\begin{array}{c}\text { 学生分组完成的大 } \\
\text { 作业 }\end{array}$ & 小组作业评分标准 \\
\hline \multirow{4}{*}{$\begin{array}{l}\text { 目标3. 能够应用数 } \\
\text { 学、自然科学和C语言 } \\
\text { 基本原理对相关的复 } \\
\text { 杂工程问题进行表达、 } \\
\text { 描述和定义 }\end{array}$} & 考试20\% & $\begin{array}{c}\text { 课程相关知识点掌 } \\
\text { 握程度 }\end{array}$ & 试题评分标准 \\
\hline & 单元测试20\% & $\begin{array}{c}\text { 期中单元测试和期 } \\
\text { 末单元测试 }\end{array}$ & PTA测试评分标准 \\
\hline & 平时测试 $40 \%$ & 每周测试 & PTA测试评分标准 \\
\hline & 小组作业 $20 \%$ & $\begin{array}{c}\text { 学生分组完成的大 } \\
\text { 作业 }\end{array}$ & 小组作业评分标准 \\
\hline
\end{tabular}

表2 PTA测试成绩评定办法

\begin{tabular}{|l|l|}
\hline \multicolumn{1}{|c|}{ 评价项目 } & \multicolumn{1}{|c|}{ 成绩评定依据 } \\
\hline 单元测试 & $\begin{array}{l}\text { 成绩采用系统即时评测。所有函数题和编程题都会设置多个评测点, 每个评 } \\
\text { 测点都有对应固定分值。学生提交代码根据评测点输入对应的输出是否正确 } \\
\text { 进行评判, 正确得此评测点分值, 不正确此评测点得分为0。 }\end{array}$ \\
\hline 平时测试 & $\begin{array}{l}\text { 成绩采用系统即时评测。所有函数题和编程题都会设置多个评测点, 每个评 } \\
\text { 测点都有对应固定分值。学生提交代码根据评测点输入对应的输出是否正确 } \\
\text { 进行评判, 正确得此评测点分值, 不正确此评测点得分为 } 0 \text { 。 }\end{array}$ \\
\hline
\end{tabular}


表3 小组作业评分标准

\begin{tabular}{|c|c|c|c|c|c|c|c|}
\hline \multirow{2}{*}{ 评价项目 } & \multirow{2}{*}{ 评分说明 (A级标准) } & \multirow{2}{*}{$\begin{array}{l}\text { 分 } \\
\text { 值 } \\
\end{array}$} & \multicolumn{5}{|c|}{ 评分值与评分段 } \\
\hline & & & A & $\mathrm{B}$ & $\mathrm{C}$ & $\mathrm{D}$ & $\mathrm{E}$ \\
\hline \multirow{6}{*}{ 小组参考 } & 按要求准时提交作业 & 10 & 10 & 9 & 8 & 7 & $\leq 6$ \\
\hline & 作业文档格式正确 & 10 & 10 & 9 & 8 & 7 & $\leq 6$ \\
\hline & 数据类型描述清晰, 算法思路表述清晰 & 20 & 20 & 18 & 16 & 14 & $\leq 12$ \\
\hline & 源代码运行正确, 关键代码给出注释 & 30 & 30 & 27 & 24 & 21 & $\leq 18$ \\
\hline & $\begin{array}{c}\text { 测试用例完备, 对时间测试正确, 对时间开 } \\
\text { 销分析正确, 并能提出优化方案 }\end{array}$ & 20 & 20 & 18 & 16 & 14 & $\leq 12$ \\
\hline & $\begin{array}{c}\text { 程序调试过程出现错误分析及调试方法有 } \\
\text { 可借鉴性 }\end{array}$ & 10 & 10 & 9 & 8 & 7 & $\leq 6$ \\
\hline
\end{tabular}

\section{4. 结束语}

移动互联网和新一代信息技术的快速发展，都使得教育资源的获取、教学手段和工具的 使用变得更加多样化, 利用优秀的互联网教育资源和信息化教学工具开展混合式教学模式是 必然的选择。同样, 随之对应的学生的学习评价方案也应该进行改变。评价目标和评价项目 要多样化, 学生成绩应该由整个学习过程中所有的有效成绩组成, 从知识目标、能力目标、 素养目标等多方面考察学生。评价主体也应该多元化, 既有系统评测, 也有教师评价, 还有 学生互评等。

\section{致谢}

本文为临沂大学学生学习评价改革课程《数据结构》、教学信息化研究项目《SPOC+在 线评测 + 雨课堂的智慧式混合教学模式在数据结构课程中的研究与应用》和教学研究与改革项 目《基于在线评测和项目导向的 $\mathrm{VC}++$ 应用开发教学模式研究》的阶段性成果之一，同时受到 山东省本科高校教学改革研究项目《地方高校基于校企协同的新工科专业改造升级探索与实 践-以软件工程为例》（M2018X058）的资助。

\section{References}

[1] Chen Yefang and Hu Wang Xiaoli, Online Practice of Programming Course in Blended Teaching Model, Journal of NingBo University, vol.41.1, pp. 103-107, 2019.

[2] Li Ling and Chen Chao, The Practice of Flipping Classroom Teaching of Science and Technology Information Retrieval Course on Rain Classroom, Library and Information Service, vol. 63.12, pp. 66-71, 2019.

[3] Li Xia, Qi Xuedong and Zhang Dongzhi etc, Integration of Internet+ Micro-Learning Evaluation Platform Construction, Jouranal of EEE,vol.41.1, pp. 41-43, 2019.

[4] Shang Chaowang, Han Meng and Liu Qingtang etc, A Study on the Model of the Creative Education Supports Students' Core Literacy, Leaning Analysis and Education Evaluation, vol. 1.5, pp. 90-95, 2018. 